\title{
Set Visualisations with Euler and Hasse Diagrams
}

\author{
Uta Priss ${ }^{(凶)}$ \\ Zentrum für erfolgreiches Lehren und Lernen, Ostfalia University, Wolfenbüttel, Germany \\ http://www. upriss.org.uk
}

\begin{abstract}
This paper discusses set visualisations with concept lattices in the sense of Formal Concept Analysis (FCA) in contrast to visualisations with Euler diagrams. Both types of visualisations have advantages and disadvantages. Because of the connection between both fields and the body of knowledge that exists in both fields it is of interest to investigate whether results from either field can contribute to the other.
\end{abstract}

\section{Introduction}

Sets and their intersections can be visualised with Venn and Euler diagrams but also using mathematical lattice theory and a certain type of diagram (Hasse diagram) that is commonly used with lattices. It is therefore of interest to compare Euler and Hasse diagrams both with respect to what can be observed from the diagrams but also with respect to underlying theoretical constructs. While a translation between lattices and Venn diagrams is straightforward, the connection between well-formed Euler diagrams and lattices is not trivial. Lattice theory has produced a large body of knowledge which could potentially be beneficial for research about well-formed Euler diagrams. The research about Venn and Euler diagrams provides, for example, applications and algorithms which could be of interest for Hasse diagrams as well.

The version of lattice theory used in this paper is called Formal Concept Analysis (FCA) and has been developed since the 1980s as an applied mathematical theory of knowledge representation (Ganter and Wille 1999). Venn and Euler diagrams are wellestablished as a visualisation of sets that is used, for example, in schools when students are first introduced to set theory. Hasse diagrams may be less intuitive at first sight and require some training. Priss (2017) discusses misconceptions that students initially have about Hasse diagrams of concept lattices in general. If restricted to specific tasks, Eklund et al. (2004) show, however, that novice users can be instructed to use Hasse diagrams fairly effectively.

As far as we know, the relationship between FCA and Euler diagrams has so far not been investigated in any great depth ${ }^{1}$. The intention of this paper is to elaborate the basic connections between both fields. This paper provides an introduction to both fields and basic translations between Venn/Euler and Hasse diagrams. It discusses the application of some lattice-theoretical properties to Euler diagrams. We suspect that

\footnotetext{
${ }^{1}$ As evidenced by a query on Google Scholar for "Formal concept analysis" and "Euler diagrams" which retrieves very little.
} 
many researchers from either field are not aware of all of the connections. Because each field has a slightly different focus, it is conceivable that a combination might provide further interesting results. Many questions about the relationship between well-formed Euler diagrams and lattices still remain open.

Sections 2 and 3 of this paper provide introductions to Venn, Euler and Hasse diagrams and FCA. Section 4 covers Venn diagrams and their (well-known) relationship to Boolean lattices. Sections 5, 6, 7 discuss different aspects of the relationship between Euler and Hasse diagrams. Although most of the individual mathematical aspects presented in this paper are not new, we believe that the compilation and elaboration of details with respect to the examples presented in this paper is new. A possibly provocative conclusion of this paper is that although many people may find Euler diagrams "intuitive" as a representation of sets, from a structural viewpoint Hasse diagrams are potentially more suitable for visualising set theory than Venn and Euler diagrams.

\section{A Brief Introduction to Euler and Venn Diagrams}

Venn and Euler diagrams are a means for graphically representing sets and their intersections and unions. A more detailed introduction and further background is, for example, provided by Rodgers (2014). Venn diagrams contain all possible intersections for a powerset (i.e. set of all subsets of a set). For example, D1 and D2 in Fig. 1 show Venn diagrams for 3 and 4 sets. Venn diagrams for more than 3 sets cannot be represented by only using circles. Euler diagrams are similar to Venn diagrams but exclude zones which are known to be empty.

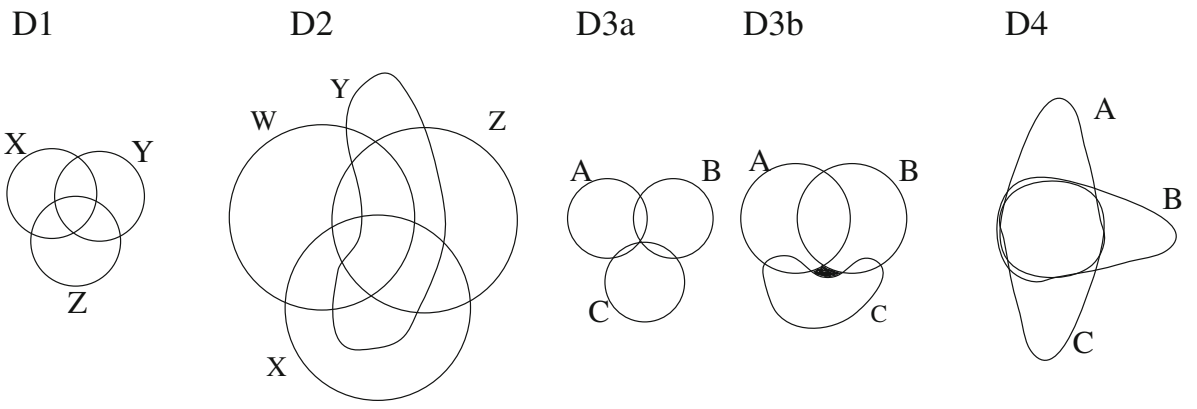

Fig. 1. Venn diagrams (D1 and D2) and non-well-formed Euler Diagrams (D3a, D3b and D4)

The following terminology applies to Venn and Euler diagrams in this paper: Venn and Euler diagrams consist of closed curves which have labels. Minimal regions are the smallest areas in a diagram which are surrounded by lines and not divided further. Regions are sets of minimal regions. Zones are maximal regions that are within a set of curves and outwith the remaining curves. For a set $L$ of curve labels, the notation $E(L)$ is used in this paper for a set of zones. In other words, $E(L)$ is a subset of the powerset of $L$ that corresponds to the zones of an Euler diagram. 
The reason for distinguishing minimal regions and zones is that zones are the smallest set-theoretically meaningful areas in a diagram whereas minimal regions are the smallest visible areas in a diagram. In a well-formed Euler diagram, zones correspond to minimal regions. Further conditions for being well-formed are defined slightly differently by different authors (e.g. Flower et al. (2008)). In order to be well-formed, a diagram should not contain a zone that is disconnected and split into several minimal regions (as in D3b in Fig. 1 where the black region in the middle belongs to the outer region). Diagrams should not contain $n$-points for $n>2$ that is points where more than 2 curves cross (as in D3a). Different curves should not be concurrent (as in D4). Each curve should have at most one label. Curves should not intersect themselves. There should not be any brushing points where several curves meet without crossing.

In universal algebra or algebraic logic, relationships are established between equational classes and algebraic structures. For example, the powerset of a set with operations $\cap, \cup$ and complementation corresponds to a Boolean algebra or Boolean lattice which can be defined as an equational class. Any subset of a powerset that is closed under $\cap$ and $\cup$ corresponds to a distributive lattice which can also be defined as an equational class. While a single (fairly simple) equation is needed to determine whether a lattice is distributive, no similar simple equation or property has yet been found that determines whether a set $E(L)$ can be represented as a well-formed Euler diagram. Although it seems visually clear what Euler diagrams are and what they look like, from an algebraic viewpoint well-formed Euler diagrams are neither simple nor intuitive. So far algorithms have been provided for deciding whether an Euler diagram is wellformed (for example, Flower et al. (2008)) but not an equational characterisation.

\section{Formal Concept Analysis and Hasse Diagrams}

A brief introduction to FCA is included here. More details can be found in the main FCA textbook by Ganter and Wille (1999). FCA is a theory of knowledge representation that was invented by Rudolf Wille in the 1980s. It provides a mathematical model for conceptual hierarchies using lattice theory. A formal context is a triple $(O, A, I)$ consisting of a set $O$ of formal objects, a set $A$ of formal attributes and a binary relation $I$ between them. This paper is only concerned with finite sets. The relation $o I a$ is read as "object $o$ has attribute $a$ ". The qualifier "formal" is used because being an object or attribute is a role. The qualifier can be omitted if it is clear what is meant. Formal objects and attributes need not be "real world" objects and attributes in any sense. The left-hand side of Fig. 2 shows an example of a formal context with types of animals as formal objects and "female", "juvenile" and "male" as formal attributes. The righthand side shows a concept lattice (as defined below) using a visualisation for partially ordered sets called Hasse diagram.

Concepts are formed by starting with a set of objects, then collecting all attributes which they have in common and then adding any further objects that also have these attributes. Dually, one can also start with attributes. Formally, all common attributes of a set $O_{1} \subseteq O$ of objects are denoted by $O_{1}^{\prime}:=\left\{a \in A \mid o I a\right.$ for all $\left.o \in O_{1}\right\}$. All common objects of a set $A_{1} \subseteq A$ of attributes are denoted by $A_{1}^{\prime}:=\{o \in O \mid$ $o I a$ for all $\left.a \in A_{1}\right\}$. A formal concept is a pair $\left(O_{1}, A_{1}\right)$ where $O_{1}=A_{1}^{\prime}$ and $A_{1}=$ 


\begin{tabular}{|l|c|c|c|}
\cline { 2 - 4 } \multicolumn{1}{l|}{} & juvenile & male & female \\
\cline { 2 - 4 } \multicolumn{1}{|c|}{ lamb } & $\mathrm{x}$ & & \\
\hline filly & $\mathrm{x}$ & & $\mathrm{x}$ \\
\hline colt & $\mathrm{x}$ & $\mathrm{x}$ & \\
\hline calf & $\mathrm{x}$ & & \\
\hline foal & $\mathrm{x}$ & & \\
\hline
\end{tabular}

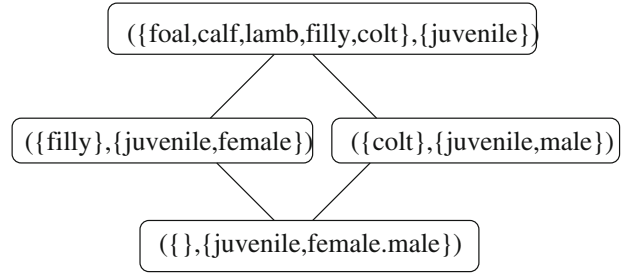

Fig. 2. A formal context and concept lattice

$O_{1}^{\prime}$. The right-hand side of Fig. 2 shows 4 formal concepts. The set $O_{1}$ of a formal concept $\left(O_{1}, A_{1}\right)$ is called the concept's extension; the set $A_{1}$ is called the concept's intension. For example, $(\{$ filly $\}$, $\{$ juvenile, female $\})$ is a formal concept with extension $\{$ filly $\}$ and intension $\{$ juvenile, female $\}$. The pair ( $\{$ calf, lamb $\},\{$ juvenile $\})$ is not a formal concept because it fulfils $O_{1}^{\prime}=A_{1}$ but not $A_{1}^{\prime}=O_{1}$. It follows from the definition of the '-operation that for any set $S$ of objects or attributes $S^{\prime}=S^{\prime \prime \prime}$ and $S \subseteq S^{\prime \prime}$.

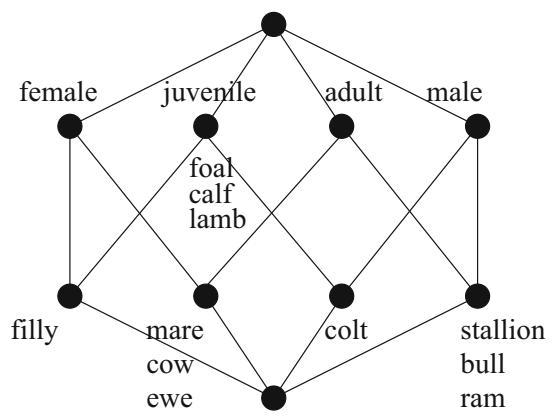

Fig. 3. A concept lattice with minimal labelling

A concept $\left(O_{1}, A_{1}\right)$ is a subconcept of a concept $\left(O_{2}, A_{2}\right)$ if $O_{1} \subseteq O_{2}$. This is equivalent to $A_{1} \supseteq A_{2}$ (as can be observed in Fig. 2). The set of formal concepts together with a subconcept ordering forms a mathematical lattice. In a Hasse diagram of a concept lattice, nodes denote concepts and edges connect adjacent concepts according to the subconcept ordering. In Fig. 2 the full concepts are written within the nodes. Figure 3 shows a different concept lattice, this time with minimal labelling because each object is written slightly below the lowest concept it belongs to and each attribute is written slightly above the highest concept it belongs to. Such objects/attributes are called immediate objects/attributes of a concept in this paper. In the remainder of this paper only minimal labelling is employed. An extension can then be read by collecting all objects on every downwards path from a concept and an intension by collecting all attributes on every upwards path from a concept. The top concept of a lattice has all objects in its extension. It can but does not need to have an attribute in its intension 
and represents some sort of global or universal concept. The bottom concept has all attributes in its intension and corresponds to some sort of Null concept. It can but does not need to have an object in its extension.

In a finite lattice, each set of concepts has an infimum (called meet and denoted by $\wedge$ ) and a supremum (called join and denoted by $\vee$ ). A meet is the largest shared concept below a set of concepts. Dually, a join is the smallest shared concept above a set of concepts. A concept in a lattice that has exactly one adjacent upper concept (i.e., one edge going up from the node) is called $\wedge$-irreducible and must have at least one immediate attribute. This is the case for all nodes that have immediate attributes in Figs. 2 and 3 except for the top concept in Fig. 2 (with attribute "juvenile") because a top concept is the meet of an empty set and thus $\wedge$-reducible. Dually, a concept with exactly one adjacent lower concept is called $\vee$-irreducible and must have at least one immediate object. In Fig. 3 the concept with immediate objects $\{$ foal, calf, lamb $\}$ is $\checkmark$-reducible. If the objects $\{$ foal, calf, lamb $\}$ were removed from the formal context the resulting lattice would still be isomorphic to the one in Fig. 3. But if "filly" or "colt" were removed from the formal context, then the lattice structure would change.

For concept lattices, logical implications amongst attributes can be read from the Hasse diagram because the attributes of a subconcept imply the attributes of a superconcept. For example, in Fig. 2 "male $\Longrightarrow$ juvenile" and in Fig. 3 "female $\wedge$ male $\Longrightarrow$ juvenile $\vee$ adult". It should be cautioned that all statements about concepts and implications are only valid for the formal context to which they belong. For example, "male $\Longrightarrow$ juvenile" is true for Fig. 2 but not for Fig. 3 .

\section{Venn Diagrams and Boolean Lattices}

Sets naturally have an extensional description by listing elements and an intensional description using logical expressions, for example consisting of labels of other sets together with set-theoretical operations. Thus, one can build formal contexts $(U, L, \in)$ where the formal objects are elements of a (universal) set $U$, the formal attributes are labels (in $L$ ) corresponding to subsets of $U$ and the incidence relation is the element-of relation $(\in)$. The Hasse diagrams below are to be interpreted in that manner. For Venn (or Euler) diagrams only set labels are required, set elements are optional but can be written into zones. In some of the Venn (and Euler) diagrams below, set elements are included in order to emphasise the correspondence between Venn and Hasse diagrams.

In a concept lattice of a context $(U, L, \in)$, the lattice-theoretical $\wedge$-operation correlates with a $\cap$-operation amongst subsets of $U$. For example in Lattice 1 in Fig. 4 , $(\{a, b\},\{X\}) \wedge(\{a, c\},\{Y\})=(\{a\},\{X, Y\})$ corresponds to $\{a, b\} \cap\{a, c\}=$ $X \cap Y$. In such lattices, the lattice-theoretical $\vee$-operation correlates with a $\cap$-operation amongst subsets of $L$. For example, in Lattice $2,\{Y, Z\} \cap\{Y, W\}=\{Y\}$. In either case, only containment holds for $\cup$-operations. For example, $(\{F, B, a\},\{Y, Z\}) \vee$ $(\{G, B, a\},\{Y, W\})=(\{F, G, B, a, l\},\{Y\})$ but $(Y \cap Z) \cup(Y \cap W) \subset Y$.

Lattices corresponding to Venn diagrams (Fig. 4) are Boolean lattices and contain $2^{n}$ concepts (for $n$ labels) each of which relates to a zone in a Venn diagram. Their Hasse diagrams form hypercubes. The dotted lines in Lattice 2 correspond to zones in Diagram 2 that are not neighbours in the Venn diagram even though they could 


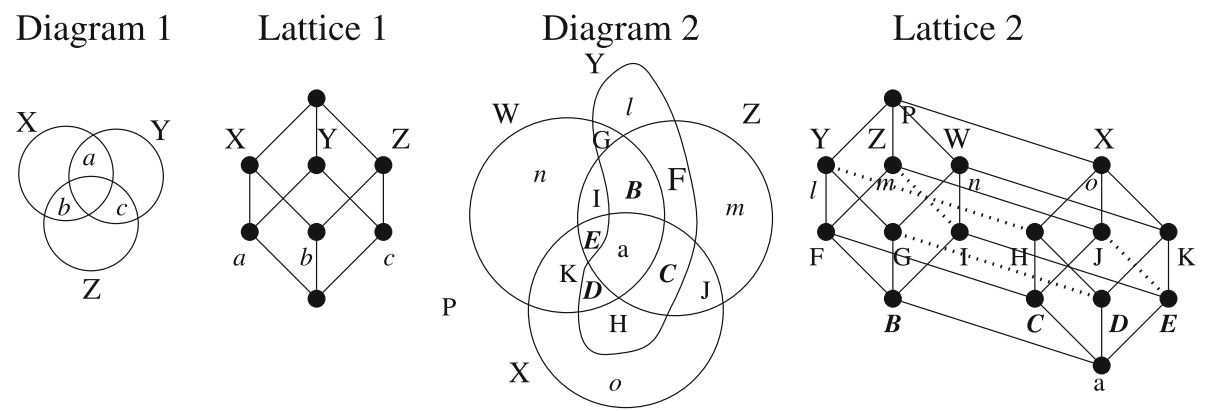

Fig. 4. Venn and Hasse diagrams of Boolean lattices

(or should) be neighbours. For example: the zone with the immediate object $H$ is in $X \cap Y$. But while it is a neighbour of the zone with the immediate object $o$ (in $X$ ) it is not a neighbour of the zone with the immediate object $l$ (in $Y$ ) even though structurally the relationship between $X$ and $X \cap Y$ is isomorphic to the relationship between $Y$ and $X \cap Y$. Thus Lattice 2 shows relationships which are not as easily visible in Diagram 2 .

Flower et al. (2008) define a dual graph of an Euler diagram as a labelled graph which has a vertex for each zone and an edge if the zones are neighbours. Each edge is labelled by the set labels which distinguish their vertices. For example, an edge between $X$ and $X \cap Y$ is labelled by $Y$. Flower et al. show that for well-formed Euler diagrams, each edge has exactly one label. This condition is called single-label condition in the remainder of this paper. A superdual graph contains all possible edges with exactly one label. Thus the dual graph of Diagram 2 corresponds to the solid lines in the diagram for Lattice 2 (as an undirected graph) whereas the superdual graph corresponds to the solid together with the dotted lines. A superdual graph represents an abstract set of zones of an Euler diagram that is independent of how the diagram is exactly drawn. The next section shows that not every abstract set of zones of a well-formed Euler diagram forms a lattice and not every lattice corresponds to a set of zones of a well-formed Euler diagram.

\section{Sets of Zones as Well-Formed Euler Diagrams and Lattices}

In this section a different construction is used for the formal contexts compared to the previous section. For each Euler diagram, a formal context $(E(L), L, \ni)$ is created by taking the set $E(L)$ of the set of labels of each zone as formal objects, the set $L$ of set labels as formal attributes and by defining the incidence relation for $z \in E(L), l \in L$ as follows: $z \ni l: \Longleftrightarrow l$ is an element of the set $z$ of labels. Graphically this is equivalent to $\mathrm{z}$ (as a zone) being within curve $l$. Contrary to the construction of $(U, L, \in)$ in the previous section this construction uses zones represented by labels without specifying elements of the sets.

The question arises as to whether any given set of zones $E(L)$ can be represented as a well-formed Euler diagram or a Hasse diagram of a concept lattice. Obviously, the condition for being representable as a concept lattice is that the set of zones must 
form a lattice. This means that in the context $(E(L), L, \ni)$ the set $E(L)$ must be closed with respect to intersections. If the set of zones itself does not form a lattice, it can still be embedded into a lattice. Constructing a concept lattice for a context $(E(L), L, \ni)$ achieves such an embedding. In the remainder of this paper, any concept in the lattice of $(E(L), L, \ni)$ that is added for the embedding (i.e. does not have an immediate object in the lattice of $(E(L), L, \ni))$ is represented by an empty node in the Hasse diagram and called a supplemental concept. Because it does not correspond to a zone and thus does not have an immediate object, the extension of a supplemental concept equals the union of the extensions of its lower neighbouring concepts. If the bottom concept is supplemental (as in Lattice 3 in Fig. 5), its extension is empty.

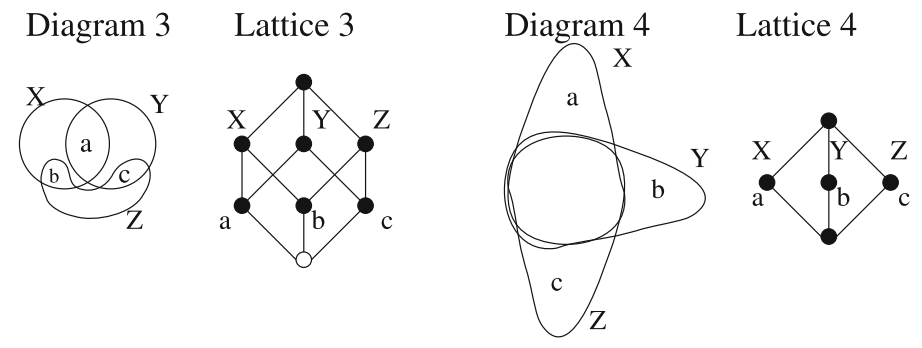

Diagram 5 Lattice $5 \quad$ Diagram $6 \quad$ Lattice $6 \quad$ Diagram 6a
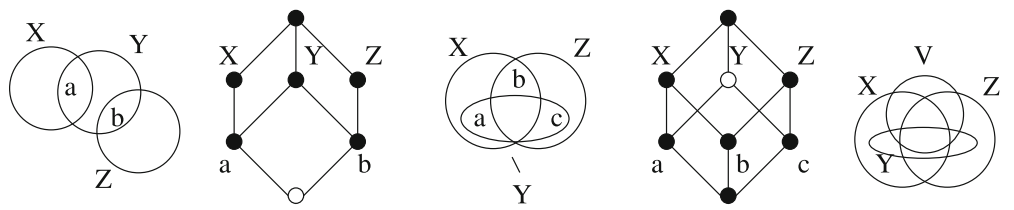

Fig. 5. Euler diagrams and concept lattices

Diagram 1 (in Fig. 4) presents a Venn diagram that is also a well-formed Euler diagram and can be represented as a lattice without supplemental concepts (cf. Lattice 1). Lattice 3 and Diagram 3 (in Fig. 5) represent a set of zones which can neither be a wellformed Euler diagram nor a lattice without supplemental concepts. The set of zones in Lattice 4 forms a lattice without supplemental concepts, but does not correspond to a well-formed Euler diagram (because it contradicts the single-label condition). Last but not least, Diagram 5 displays an example of a well-formed Euler diagram which does not correspond to a lattice without supplemental concepts. Thus the examples show that any of the four possible constellations of being a well-formed Euler diagram and a lattice without supplemental concepts exists.

Supplemental concepts can occur higher up in the lattice ordering as well. Lattice 6 contains a supplemental concept which is required in order to attach attribute $Y$ to a node but this node does not correspond to a zone in Diagram 6. Lattice 6 would still be a lattice even if the supplemental concept was removed. But in that case instead of a curve $Y$, two curves would need to exist, one as a subset of $X$ and the other one 
as a subset of $Z$. Thus a corresponding lattice without a supplemental concept would have one attribute more than Lattice 6. Its corresponding Euler diagram would not be well-formed because the single-label condition would not be fulfilled.

It should be mentioned that adding or deleting a curve can change a well-formed Euler diagram into a non-well-formed one and vice versa. Diagram 6 can be embedded into a well-formed Euler diagram by adding a curve as shown in Diagram 6a. Similarly in Diagram 2, deleting curve $W$ or $X$ would yield a non-well-formed diagram which, in this case however, can be transformed into a well-formed diagram. For the purposes of this paper this fact about Euler diagrams is stated as the set of well-formed Euler diagrams not being closed with respect to recursive generation.

\section{Conditions for Well-Formed Euler Diagrams}

It appears to be easier to identify conditions that determine that a set of zones cannot be a well-formed Euler diagram than those that determine that it can be a well-formed Euler diagram. Such conditions from the literature (see below) tend to not use lattice theory. Therefore this section discusses some conditions based on lattice theory.

- C1: if a $\wedge$-reducible concept has an immediate attribute then the corresponding Euler diagram is not well-formed. Proof: a curve corresponding to such an attribute is concurrent with the intersection of other curves which contradicts the single-label condition.

- C2: if a $\wedge$-reducible concept with $n$ adjacent upper concepts in a concept lattice without supplemental concepts is not the bottom node of a Boolean sublattice with $2^{n}$ elements, then the corresponding Euler diagram is not well-formed. Proof: if a concept $c$ has $n$ adjacent upper neighbours then the intensions of these concepts must all differ by one attribute from the intension of $c$ because of the single-label condition. This means that any pair of the $n$ concepts shares all attributes except that each one has one extra attribute. If one forms all intersections of such $n$ intensions in order to build a lattice without supplemental concepts then one obtains a Boolean sublattice.

Condition $\mathrm{C} 2$ is relevant for Lattice 4 and the discussion about Lattice 6 above. Because of condition $\mathrm{C} 2$, lattices without supplemental concepts that correspond to well-formed Euler diagrams look like they are hypercubes that are glued together. But this is still not a necessary and sufficient condition. Lattice 9 in Fig. 6 does not correspond to a well-formed Euler diagram because the zone $\{X, W\}$ which is shaded in black is disconnected.

A next attempt might be to consider whether distributivity plays a role but Fig. 6 demonstrates that it does not. Lattice 7 is not distributive but Diagram 7 is well-formed. Lattices 8-10 are distributive. Lattices 8 and 10 can be represented as well-formed Euler diagrams (as shown in Diagrams 8 and 10) but Lattice 9 cannot. In the case of a single disconnected zone as in Diagram 9, adding a further zone yields a well-formed diagram as demonstrated for Diagram 6 and 6a, Diagram 9 and 10 and Diagram 5 (modified to correspond to a lattice without supplemental concepts) and Diagram 7. Each represents 


\section{Diagram 7}

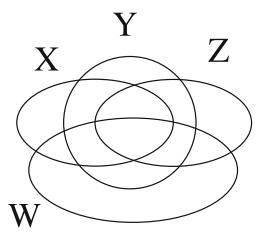

Lattice 7

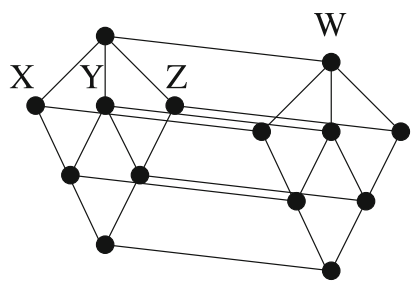

Diagram 8

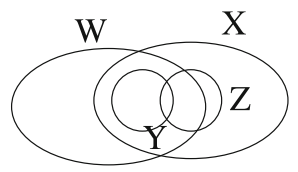

Diagram 9

Diagram 10
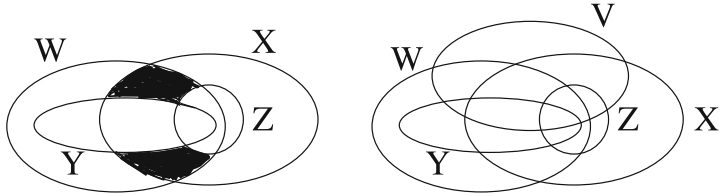

Lattice 8

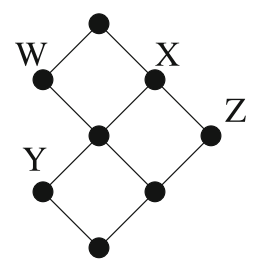

Lattice 9

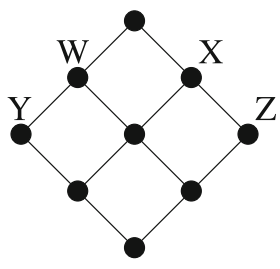

Lattice 10

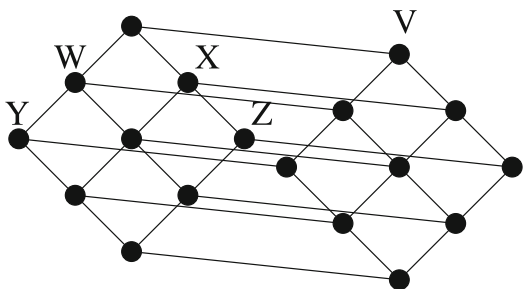

Fig. 6. Euler diagrams and distributive lattices

an example of the set of well-formed Euler diagrams not being closed with respect to recursive generation.

The fact that Lattices 4 and 9 cannot be represented as well-formed Euler diagrams can also be described in terms of irreducible concepts (i.e. concepts that are simultaneously $\wedge$ - and $\vee$-irreducible): in Lattice 4 a meet of three irreducible concepts contradicts the single-label condition and in Lattice 9 two irreducible concepts that have pairwise meets with the same concept and a joined meet cause a similar problem. It might also be of interest to consider well-formed Euler diagrams for lattices such as Lattice 5 where only the bottom concept is a supplemental concept.

Flower et al. (2008) provide further necessary conditions for well-formed Euler diagrams, for example a connectivity condition: a dual graph serves the connectivity condition if it is connected, all subgraphs induced by deleting any vertex containing a selected label are connected and all subgraphs induced by deleting any vertex not containing a selected label are also connected. If the bottom node was missing in Lattice 6 , then its graph would be disconnected after removal of all concepts which do not contain $Y$. Thus attributes attached to supplemental concepts can be necessary, but not sufficient for the connectivity condition. 
A further condition from Flower et al. (2008) is that the dual graph of an Euler diagram must be planar or must be reducible to a planar graph which still passes the connectivity condition. This does not imply that the corresponding Hasse diagram also must be planar because a Hasse diagram is a directed graph whereas a dual graph is undirected. For example Lattice 1 in Fig. 4 is not planar and cannot be converted into a planar Hasse diagram. But if the graph is converted into an undirected graph and the top node (or the bottom node) is placed into the middle then it can be drawn as a planar graph. The same holds for Lattice 2 without the dotted lines. Again, the negation is not valid: Lattice 9 shows an example that fulfils the single-label condition, the connectivity condition and is a planar Hasse diagram but is not drawable as a well-formed Euler diagram. Flower et al. remaining condition is a "face condition" which checks the sequence of curve labels around each "face" of a dual graph for a certain property. It is not clear whether and how that could be translated into a lattice-theoretical property.

\section{Reading Implications from Euler and Hasse Diagrams}

The question of which Euler diagrams can be drawn as well-formed diagrams is important because well-formed diagrams are presumably easier for users to visually parse than non-well-formed diagrams. A further question about Euler diagrams is what information can be extracted from them so that they can be employed as a tool for information visualisation. In Sect. 3 it was mentioned that implications can be read from concept lattices. The same is true for Euler diagrams. For example, one can read $X \Longrightarrow Y$ and $Y \Longrightarrow Z$ both from Diagram 11 as well as from Lattice 11 (in Fig. 7).

Stapleton et al. (2017) use Diagram 12 as an example of an observational advantage of Euler diagrams. The diagram shows that $P \cap Q=\emptyset \Rightarrow R \cap Q=\emptyset$. Stapleton et al. argue that Euler diagrams have a maximum observational advantage because any similar set-theoretical statement that is valid for the data in the diagram can be read from the diagram. We argue that Hasse diagrams have an even higher observational advantage than Euler diagrams if one considers further set-theoretical operations.

The implication $P \cap Q=\emptyset \Rightarrow R \cap Q=\emptyset$ can also be observed from Lattices $12 \mathrm{a}$ and $12 \mathrm{~b}^{2}$. Lattices $12 \mathrm{a}$ and $12 \mathrm{~b}$ both contain the implication $R \Rightarrow P$ and the corresponding $R \cap Q \subseteq P \cap Q$. Lattice 12a also contains $P \cap Q \Rightarrow R$ and thus $P \cap Q=R \cap Q$ which is difficult, or impossible, to see in Diagram 12 because it involves a statement about the empty set as a bottom concept which exists in Lattice 12a but is a missing zone in Diagram 12. Lattice 12b contains all intersections that are still possible if the implication $R \Rightarrow P$ is assumed. The supplemental concepts in Lattice $12 \mathrm{~b}$ correspond to two missing zones in Diagram 12. In Lattice 12b, the implication $P \cap Q=\emptyset \Rightarrow R \cap Q=\emptyset$ is not an intensional implication but an implication that involves $R \cap Q \subseteq P \cap Q$ and the extensional information that $P \cap Q=\emptyset$.

While it is possible to observe that zones are missing in an Euler diagram, one can argue that statements that assert that two missing zones are equal (as in Lattice 12a) or involve information about extensions (as in Lattice 12b) cannot be observed from Euler diagrams. Thus one might argue that for someone who can read Hasse diagrams,

\footnotetext{
${ }^{2}$ It should be noted that implications and their generalisations are well-known in the FCA community and discussed, for example, in the textbook by Ganter and Obiedkov (2016).
} 
Lattices $12 \mathrm{a}$ and $12 \mathrm{~b}$ have a higher observational advantage than Diagram 12. Furthermore, Hasse diagrams are not restricted to representing simple relationships amongst sets. Ganter and Obiedkov (2016) discuss many other applications, for example, involving clauses and other more complex logical statements instead of just implications.

It should be mentioned, however, that lattices have the same problem as Venn and Euler diagrams in that they become very difficult to visually parse if they are too large. In cases such as Lattice 4 in Fig. 5 where "many intersections are missing", the lattice is less complex than a Boolean lattice. But in cases such as Lattice 3, a Boolean lattice is required. While it is theoretically possible to draw Hasse diagrams for Boolean lattices of any size, it becomes difficult to see anything in such a lattice for more than 4 sets. Therefore presenting diagrams to users is not necessarily the main goal of FCA applications which instead often use FCA for computational purposes.

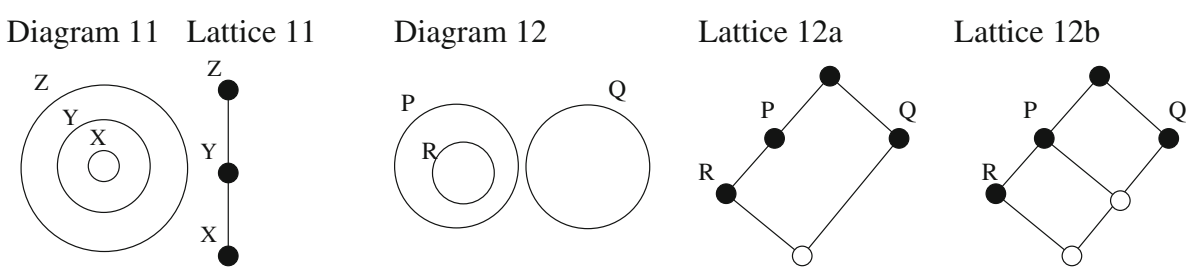

Fig. 7. Implications amongst set-theoretical statements

\section{Conclusion}

This paper provides a discussion of representing sets with Hasse diagrams of concept lattices compared to Euler diagrams. The basic relationship between the two types of diagrams is explained. Examples of well-formed Euler diagrams exist that do not correspond to lattices without supplemental concepts and lattices without supplemental concepts exist that do not correspond to well-formed Euler diagrams. Conditions for determining which Euler diagrams can be represented as concept lattices without supplemental concepts are discussed. While some Euler diagrams that are not well-formed can be near impossible to draw, having supplemental concepts in a lattice does not affect how a Hasse diagram is drawn or read. Supplemental concepts serve a purpose with respect to implications. Both Euler diagrams and Hasse diagrams become difficult to read if they get too large. While many people find Euler diagrams much more intuitive to read than Hasse diagrams, the overall expressive power of Hasse diagrams might be higher than that of Euler diagrams. Furthermore, lattice theory can quite likely provide more insights with respect to a theory of well-formed Euler diagrams.

One potentially provocative conclusion of this paper is that well-formed Euler diagrams may not actually be an ideal representation for sets. Set theory is often introduced to students using the visualisation of Venn and Euler diagrams. Thus students may start to think of sets as being like Venn and Euler diagrams. But because well-formed Euler 
diagrams can only represent some subsets of powersets and because it is not clear what the algebraic nature of well-formed Euler diagrams precisely is, one could argue that in some sense Hasse diagrams are more suitable for representing set theory than Euler diagrams.

\section{References}

Eklund, P., Ducrou, J., Brawn, P.: Concept lattices for information visualization: can novices read line-diagrams? In: International Conference on Formal Concept Analysis, pp. 57-73. Springer, Berlin (2004)

Flower, J., Fish, A., Howse, J.: Euler diagram generation. J. Vis. Lang. Comput. 19(6), 675-694 (2008)

Ganter, B., Wille, R.: Formal Concept Analysis. Mathematical Foundations. Springer, Berlin (1999)

Ganter, B., Obiedkov, S.: Conceptual Exploration. Springer, Berlin (2016)

Priss, U.: Learning thresholds in formal concept analysis. In: Bertet, K., Borchmann, D., Cellier, P., Ferré, S. (eds.) ICFCA 2017. LNCS (LNAI), vol. 10308, pp. 198-210. Springer, Cham (2017). https://doi.org/10.1007/978-3-319-59271-8_13

Rodgers, P.: A survey of Euler diagrams. J. Vis. Lang. Comput. 25(3), 134-155 (2014)

Stapleton, G., Jamnik, M., Shimojima, A.: What makes an effective representation of information: a formal account of observational advantages. J. Logic Lang. Inform. 26(2), 143-177 (2017)

Open Access This chapter is licensed under the terms of the Creative Commons Attribution 4.0 International License (http://creativecommons.org/licenses/by/4.0/), which permits use, sharing, adaptation, distribution and reproduction in any medium or format, as long as you give appropriate credit to the original author(s) and the source, provide a link to the Creative Commons license and indicate if changes were made.

The images or other third party material in this chapter are included in the chapter's Creative Commons license, unless indicated otherwise in a credit line to the material. If material is not included in the chapter's Creative Commons license and your intended use is not permitted by statutory regulation or exceeds the permitted use, you will need to obtain permission directly from the copyright holder.

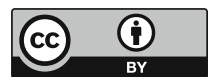

